\title{
A INFLUÊNCIA DA ORIENTAÇÃO DA ENFERMAGEM ANTES DA ALTA HOSPITALAR NO PROGNÓSTICO DO PACIENTE
}

\author{
THE INFLUENCE OF NURSING ORIENTATION BEFORE THE PATIENT'S NON- \\ PROGNOSTIC HOSPITAL HIGH
}

'Jennyfer Stefanny Paula Pereira, 'Josefa Maria de Oliveira Pinto, 'Daniel Asaph Guimarães Castro

\section{RESUMO}

Introdução: Pacientes no âmbito hospitalar recebem da enfermagem orientações e cuidados direcionados ao seu tratamento. Porém, após a alta hospitalar, o paciente não tem mais a presença do profissional para acompanha-los, de modo que, os procedimentos são realizados pelo próprio paciente ou familiar. Objetivos: Demonstrar como a orientação de enfermagem, antes da alta hospitalar, influencia diretamente no prognóstico do paciente, e indicar, baseando na literatura, maneiras corretas de realizar uma boa orientação. Materiais e Métodos: realizou-se uma pesquisa bibliográfica, qualitativa, de caráter exploratória e dedutivo. Foram utilizados materiais publicados entre os anos de 2005 à 2016, disponibilizados gratuitamente e com texto completo, e que apresentou concordância direta com o tema proposto. A análise e interpretação de dados obtidos ocorreram com base em materiais selecionados para o estudo, que posteriormente foram fixadas e organizadas de acordo com o corpo do trabalho. Discussão: a enfermagem venha aumentar suas compreensões e habilidades sobre a complexidade da situação-problema do indivíduo e de suas autênticas limitações, dificuldades, capacidades e necessidades, de maneira a assistilos a conhecer esta experiência como componentes ativos do progresso e não como meros expectadores das atividades dos profissionais de saúde e de seus cuidadores. Conclusão: foi verificado um quantitativo baixo de estudos associados com alta hospitalar, onde observamos que os estudos associados a esta temática expõem maior enfoque nas patologias dos pacientes, do que nos procedimentos de alta hospitalar ou situação-problema destes e de seus familiares propriamente ditos.

Descritores: Alta. Orientação. Enfermagem. Reinternação.

\section{ABSTRACT}

Introduction: Patients in the hospital receive nursing guidance and care directed to their treatment. However, after hospital discharge, the patient no longer has the presence of the professional to accompany them, so that the procedures are performed by the patient or family member. Objectives: To demonstrate how nursing orientation, before hospital discharge, directly influences the prognosis of the patient, and to indicate, based on the literature, correct ways to perform a good orientation. Materials and Methods: a bibliographical, qualitative, exploratory and deductive research was carried out. Materials published between the years 2005 and 2016, free of charge and with full text, were used and presented direct agreement with the proposed theme. The analysis and interpretation of data obtained were based on materials selected for the study, which were later fixed and organized according to the body of the work. Results and Discussion: Nursing will increase their understanding and skills about the complexity of the problem situation of the individual and their authentic limitations, difficulties, abilities and needs, in order to assist them to know this experience as active components of progress and not as mere Viewers of the activities of health professionals and their caregivers. Conclusion: a low number of studies associated with hospital discharge were found, where we observed that the studies associated with this topic expose a greater focus on the patients' pathologies than on the discharge procedures or the problem situation of these patients and their families.

Descriptors: High. Guidance. Nursing. Rejection

\section{INTRODUÇAOO}

Educar é uma técnica da enfermagem que tem como objetivo estabelecer planejamentos visando à recuperação da saúde. É uma das atividades que valoriza a enfermagem na modificação de atitudes perante de uma cesta patologia. Os profissionais de enfermagem na promoção da saúde estarão comprometidos a reproduzir programas, e a liderança de equipes de serviço saúde, cujos atos objetivam trabalhar para promover atividades, atitudes e condutas de saúde positivas ${ }^{1}$. 
Recebido: 31 maio 2017. Aceito: 14 dezembro 2018. Revista Amazônia Science \& Health - 2018 Out/Dez.
Pereira, JSP. Pinto, JMO. Castro DAG.

A INFLUÊNCIA DA ORIENTAÇÃO DA ENFERMAGEM ANTES DA ALTA HOSPITALAR NO PROGNÓSTICO DO PACIENTE
O planejamento da alta se tornou uma grande prioridade para doentes e familiares, e é um procedimento necessário, principalmente quando a alta pode vir a ser um problema ou quando o internamento é prolongado ${ }^{2}$.

Os pacientes no âmbito hospitalar recebem da enfermagem orientações e cuidados direcionados ao seu tratamento e necessidades baseada num plano individual e contínuo. Estes cuidados são preparados por profissionais que desempenham tal função de maneira científica. Porém, após a alta hospitalar, o paciente não tem mais a presença do profissional para acompanhalos, de modo que, os procedimentos são realizados pelo próprio paciente, familiares ou cuidadores.

No ambiente hospitalar, um bom planejamento da alta é um procedimento que visa realizar orientações profissionais. Tem a finalidade propor o autocuidado, a autonomia e acesso aos processos de saúde disponíveis, para que os usuários recuperem a saúde por meio de recursos assistenciais complexos. A alta hospitalar é a mudança dos cuidados ao paciente do hospital para o âmbito domiciliar.

Perante o surgimento de uma doença a família naturalmente é a primeira referência de apoio para a pessoa fragilizada. O plano de alta deve garantir que os cuidados terão continuidade, para que após a alta o tratamento não pare e não seja prejudicado por falta de orientações. O mesmo autor concorda ainda que, o doente e a família recebam orientações bem planejadas na alta, e bem esclarecidas ao paciente, que garantam a estes o conhecimento necessário para dar prosseguimento aos cuidados ${ }^{3}$.

No período de internação, quando o enfermeiro presta assistência ao paciente em conjunto com os familiares, e os mesmos tem a oportunidade de participar do processo de cuidar, estes acabam desenvolvendo autonomia e garantindo um bom seguimento dos cuidados, ao regressar para casa ${ }^{4}$.

A alta hospitalar $(\mathrm{AH})$ é definida como a condição que permite a partida do indivíduo do ambiente hospitalar, considerando-se todas as formas pelas quais o paciente deixa o hospital, seja com consentimento da equipe médica, vontade do próprio paciente ou ainda por óbito².

Como forma de garantia que os cuidados serão realizados corretamente após a alta hospitalar é necessário um programa de alta criado especialmente para cada paciente, de forma que este programa contribua para que a família possa desenvolver os cuidados necessários e dar continuidade no tratamento iniciado no hospital. Compreende, portanto, 0 prosseguimento do tratamento e maior qualidade de vida para o cliente ${ }^{5}$.

O programa de alta hospitalar pode, e muito, proporcionar tratamento e recuperação de forma mais humanizada e gerar bem-estar ao paciente $e$ ao cuidador ${ }^{6}$.

Para possibilitar a sequência dos cuidados que foram iniciados no hospital, o familiar ou cuidador dependerá das orientações realizadas ao mesmo a respeito da alta hospitalar, uma vez que esta irá contribuir para um melhor prognóstico do cliente, diminuindo a insegurança e proporcionando melhor qualidade de vida no âmbito familiar e social $^{5}$.

Os Planos, organizações e estrutura da alta hospitalar são instrumentos de suma importância, pois são utilizadas para que os pacientes desenvolvam o autocuidado no pós-alta e tenham a capacidade de fazê-lo de forma segura, fortalecendo o comprometimento ao tratamento, e promovendo a redução de hospitalizações. Faz-se importante centralizar ainda, a comunicação entre o profissional de Enfermagem e paciente, para reduzir a frequência de hospitalizações não planejadas e estreitar a comunicação entre o hospital e o cuidado humanizado ${ }^{7}$.

Os pacientes, no período de hospitalização, recebem atenção integral dos profissionais da equipe multiprofissional, onde todos contribuem com a melhoria do estado de saúde do indivíduo enfermo. Especialmente 0 profissional da enfermagem que permanece ao lado do paciente nas 24 horas do dia por todo o período de internação. Durante o tratamento é fundamental que os pacientes recebam informações e orientações sobre seu estado de saúde e sobre os procedimentos a eles aplicados e, no momento da alta, é crucial que o profissional da enfermagem realize orientações acerca de todos os cuidados de que o paciente necessita.

O objetivo desse trabalho consiste em demonstrar como a importância da orientação de enfermagem, antes da alta hospitalar, influencia diretamente no prognóstico do paciente, e indicar, baseando na literatura, maneiras corretas de realizar uma boa orientação mostrando os benefícios do programa "Melhor em Casa" do Ministério da Saúde.

\section{MATERIAL E METODOS}

Este texto trata-se de uma revisão bibliográfica do tipo qualitativa de caráter exploratório com objetivo de ter uma visão centrada na orientação de enfermagem no momento da alta hospitalar, abordando a importância desta para pacientes e familiares.

A abordagem bibliográfica se constitui num procedimento formal para aquisição do conhecimento sobre a realidade. Exige pensamento reflexivo e tratamento cientifico. A pesquisa procurou dados em variadas fontes, de forma direta ou indireta ${ }^{8}$. 
Recebido: 31 maio 2017. Aceito: 14 dezembro 2018. Revista Amazônia Science \& Health - 2018 Out/Dez.
Pereira, JSP. Pinto, JMO. Castro DAG.

A INFLUÊNCIA DA ORIENTAÇÃO DA ENFERMAGEM ANTES DA ALTA HOSPITALAR NO PROGNÓSTICO DO PACIENTE
A pesquisa qualitativa é utilizada com o objetivo de conseguir informações e/ou conhecimentos acerca de um problema para o qual se procura uma resposta, ou de uma hipótese que se queira comprovar, ou ainda descobrir novos fenômenos ou as relações entre eles ${ }^{9}$.

A pesquisa exploratória estabelece critérios, métodos e técnicas para a elaboração de uma pesquisa e visa oferecer informações sobre o objeto desta e orientar a formulação de hipóteses.

Os critérios de inclusão utilizados para a escolha do material bibliográfico consistiram em artigos relacionados ao tema da pesquisa. Utilizouse para a coleta dos artigos bases de dados como: Google Acadêmico; Scielo; Revista Brasileira de Enfermagem (REBEN); Medline; Ministério da Saúde (MS); Lilacs. As fontes utilizadas foram de 2005 a outubro de 2016. O critério de exclusão usado foi o de material bibliográfico fora do tema escolhido nesta pesquisa.

Para seleção dos artigos foram utilizados as palavras-chave: Alta; Orientação; Enfermagem; Reinternação. A pesquisa em Literatura de modo geral exige a aplicação de metodologia e técnicas apropriadas. O pesquisador deve voltar sua atenção para os fatos literários ou linguísticos, desprezando 0 anedótico e o individual. A realização da pesquisa cientifica exige utilização de métodos dedutivos (premissas de um raciocínio para chegar a uma conclusão) e indutivos (é um raciocínio em que, de fatos particulares, se tira uma conclusão genérica) ${ }^{8}$.

Após a seleção dos artigos foi realizado a leitura e fichamento dos artigos relacionados ao tema a orientação de enfermagem no momento da alta hospitalar, abordando a importância desta para pacientes e familiares.

As informações serão acomodadas a partir da seleção do assunto, levando em consideração o foco do presente estudo e as palavras chaves, bem como os autores, o ano da publicação, a abordagem especifica, a metodologia utilizada e a síntese da conclusão. Para tanto será feito um quadro síntese para os objetivos contendo essas informações.

Dessa maneira, oportunizando a análise das informações e comparação das abordagens teóricas que contemplam os objetivos da pesquisa que, consequentemente, facilitará que os autores possam no final do trabalho construir uma reflexão critica a partir dos estudos levantados.

\section{RESUTADOS E DISCUSSÃO}

Não foram encontrados muitos artigos que abordasse essa temática, ressaltando a necessidade de mais pesquisa para assim melhor levantar a importância da enfermagem após a alta hospitalar, porém a elaboração de orientações sistematizadas de enfermagem para alta hospitalar concluiu que materiais educativos possibilitam ao enfermeiro, dentre outros benefícios, identificar as reais necessidades dos pacientes, avaliar a compreensão de orientações de cuidados e, consequentemente, contribuir para a continuidade do tratamento em domicílio.

Orientações de enfermagem por escrito foram fornecidas apenas para 7,9\% participantes e limitaram-se às anotações quanto aos horários de administração dos medicamentos, na própria receita médica do participante ${ }^{11}$.

O mesmo autor mostrou que $33,3 \%$ da amostra apresentaram dúvidas durante a orientação final de enfermagem, sendo essas principalmente a respeito da dose e do nome dos medicamentos.

Com objetivo de verificar o planejamento da assistência de enfermagem do paciente com insuficiência cardíaca (IC) à alta hospitalar outro pesquisador destacou os aspectos relacionados à interdependência dos cuidados de enfermagem com a equipe multidisciplinar e orientações sobre 0 planejamento da assistência de enfermagem, importância dos registros e protocolos de enfermagem e outros ${ }^{13}$.

Em outra pesquisa os resultados foram semelhantes uma vez que o planejamento da alta hospitalar é o resultado do cuidado multiprofissional, devendo ser interdisciplinar, numa perspectiva transdisciplinar do cuidado para a saúde dos clientes. As subcategorias que emergiram na pesquisa trouxeram resultados interessantes sobre os conhecimentos dos enfermeiros acerca do planejamento da alta hospitalar em que foi identificado o pensamento complexo existiu na fala de apenas um dos enfermeiros. Porém os outros depoentes referiram alta hospitalar como sendo algo fragmentado, separado ${ }^{12}$.

É necessário capacitar cuidadores e familiares, no cuidado de idosos em sua residência depois da alta hospitalar. Precisa também de uma equipe interdisciplinar, que apresente como objetivo os vários fatores como a saúde, bem-estar e qualidade de vida do idoso, no seu contexto social, considerando, sobretudo sua família ${ }^{14}$.

Educar é uma técnica da enfermagem que tem como objetivo estabelecer planejamentos visando à recuperação da saúde. É uma das atividades que valoriza a enfermagem na modificação de atitudes perante de uma cesta patologia. Os profissionais de enfermagem na promoção da saúde estarão comprometidos a reproduzir programas, e a liderança de equipes de serviço saúde, cujos atos objetivam trabalhar para promover atividades, atitudes e condutas de saúde positivas ${ }^{1}$.

$\mathrm{Na}$ alta hospitalar acontece a transferência dos cuidados do paciente do hospital para outros aspectos de saúde. Estes cuidados deixam de ser científicos e passam a ser realizados pelos familiares e cuidadores ${ }^{15}$.

É importante enfatizar que a equipe de saúde precisa-se mobilize na procura de novas técnicas de 
Recebido: 31 maio 2017. Aceito: 14 dezembro 2018. Revista Amazônia Science \& Health - 2018 Out/Dez.
Pereira, JSP. Pinto, JMO. Castro DAG.

A INFLUÊNCIA DA ORIENTAÇÃO DA ENFERMAGEM ANTES DA ALTA HOSPITALAR NO PROGNÓSTICO DO PACIENTE ensino, que atendam a necessidade particular do cidadão, objetivando garantir uma administração de medicamentos segura no domicílio.

O profissional e o paciente devem constantemente avaliar seu progresso no que se refere ao processo ensino-aprendizagem, uma vez que a mudança de comportamento do paciente constitui um importante fator para a garantia da continuidade de sua assistência no domicílio ${ }^{11}$.

Após a alta hospitalar os cuidados que eram realizados pelos profissionais de enfermagem passam a ser feitos pela família ou cuidadores. Por isso, a boa orientação da equipe de enfermagem poderá contribuir para uma melhor recuperação do paciente, minimizando o sofrimento, intercorrências e trazendo maior qualidade de vida para o mesmo e a família. Dessa forma, pode-se prevenir complicações como retorno ao hospital, infecções, maior risco de morte e, consequentemente, evitar gastos hospitalares desnecessários.

É importante destacar que para evitar ocorrência de erros de medicação no domicílio o enfermeiro necessita ter conhecimento da ação do medicamento no organismo, reações colaterais, das técnicas de administração pelas diferentes vias, métodos e vias de administração, eliminação, dose máxima e terapêutica, efeitos tóxicos, assim como, da fisiologia e anatomia humana, porquanto, falhas no conhecimento da enfermagem refletem inteiramente na orientação que é transmitida ao paciente.

Foi mostrado por diversos autores que o plano de alta hospitalar precisa ser realizado pela enfermagem, mas, com a cooperação de outros profissionais que cuidam do indivíduo, procurando também melhor vínculo com as demais áreas de saúde na qual esse indivíduo encontra-se inserido resultando em um cuidado multiprofissional, devendo ser interdisciplinar e necessário capacitar cuidadores e familiares ${ }^{1}$.

Observa-se, através, desta pesquisa que a enfermagem é o profissional que elabora e gerencia e o planejamento de alta, porquanto esse profissional encontra-se consecutivamente no hospital, tornando-se uma ligação entre o paciente e os demais profissionais da equipe multiprofissional, além de ser o profissional que está mais envolvido nas pesquisas sobre planejamento de alta ${ }^{15}$.

É interessante discutir ainda, que a enfermagem venha aumentar suas compreensões e habilidades sobre a complexidade da situaçãoproblema do indivíduo e de suas autênticas limitações, dificuldades, capacidades e necessidades, de maneira a assisti-los a conhecer esta experiência como componentes ativos do progresso e não como meros expectadores das atividades dos profissionais de saúde e de seus cuidadores.

Portanto, destacamos que a obrigação dos profissionais de saúde para com o cidadão não se termine com sua saída da unidade hospitalar, sendo imprescindível que os cuidados dirigidos para estes pacientes, baseado na educação em saúde elaborada no setor de internação, para que os mantenha saudáveis no ambiente domiciliar.

\section{CONCLUSAOO}

O enfermeiro, juntamente com a equipe de saúde, organize um plano de alta individualizado para o usuário, fundamentado na sua admissão na organização, com a finalidade de garantir a continuidade da sua terapêutica no domicílio.

Uma das limitações do estudo baseia-se da busca da literatura escrita e em base de dados online foi, sobretudo verificado um quantitativo baixo de estudos associados com alta hospitalar, onde observamos que os estudos associados a esta temática expõem maior enfoque nas patologias dos pacientes, do que nos procedimentos de alta hospitalar ou situação-problema destes e de seus familiares propriamente ditos.

Em relação às últimas orientações no final da internação, ressalvamos que o indivíduo necessita recebê-las antes do horário previsto para sua saída do hospital, permitindo mais um momento para analisar sua compreensão quanto às informações recebidas, assim como permitir o esclarecimento de dúvidas, onde estas recomendações precisarão ser registradas em prontuário do usuário corretamente, conforme preconizado pela legislação de enfermagem vigente.

É importante explicar ainda, que a equipe de enfermagem venha melhorar seus conhecimentos em relação os cuidados diretos aos pacientes e suas reais limitações, necessidades, dificuldades e capacidades procurando assisti-los a vivenciar o período da internação de maneira pró-ativa e não como expectadores das atividades dos profissionais de saúde e de familiares.

\section{REFERENCIAS}

1 ECHER, I.C.; GRACIOTO, A.; GOMES, C.J.; LORENZI, P.D.C. Grupo de Orientação de Cuidados aos Familiares de Pacientes Dependentes. Revista Brasileira de Enfermagem. Jan/Fev. 2006.

\section{RAMOS, S.A. A Influência do Planejamento da Alta Hospitalar no Número de Dias de} Internamento do Doente. 2015, 76f. Dissertação (Mestrado em Gestão de Unidade de Saúde) Universidade Lusófona de Humanidade e Tecnologias, Lisboa, 2015.

3 FERREIRA, M.C.S.; GALLANI, M.C.B.J. Insuficiência cardíaca: antiga síndrome, novos conceitos e a atuação do enfermeiro. Revista 
Brasileira de Enfermagem, v. 58 n.1, p. 70-3, 2011.

4 SILVA, M.S.N. 0 acesso aos Cuidados de Enfermagem após a alta hospitalar: Uma análise do impacte da partilha de informação. $2011105 f$. Dissertação ( Mestrado em Ciências da Enfermagem) Escola Superior de Enfermagem do Porto. Cidade do Porto. 2007.

5 CESAR, A.M.; SANTOS, B.R.L. Percepção de cuidadores sobre um programa de alta hospitalar. Revista Brasileira de Enfermagem. v. 58, n. 6. p. 647-52, Nov./Dez, 2005.

6 SOUSA, V.P. A importância das orientações ao paciente hipertenso na alta hospitalar. 2014. 19 F. Monografia - Universidade Federal de Santa Catarina - Departamento de Enfermagem, Florianópolis, 2014.

8 MEDEIROS, J.B. Redação científica: a prática de fichamento, resumos, resenhas. 11 ed. São Paulo: Atlas, 2013.

9 MARCONI, M.A; LAKATOS, E.M. Metodologia do trabalho científico: procedimentos básicos, pesquisa bibliográfica, projeto e relatório, publicações e trabalhos científicos. 5. ed. São Paulo: Atlas, 2013.

10 CRUZ, I.ML.; MANTOVANI, M.F. Nursing advice for the discharge from hospital of the neoplastic patient. Cogitare Enferm., v. 19, n. 4, p. 629-35, Out/Dez, 2014

11 MIASSO, A.I.; CASSIANI, S.H.B. Administração de medicamentos: orientação final de enfermagem para a alta hospitalar. Rev Esc Enferm USP, v. 39, n. 2, p. 136-44, 2005.

12 ARAUJO, F.S.R. O planejamento da alta hospitalar pelo enfermeiro aos clientes das unidades clínicas \& cirúrgicas: perspectiva da complexidade em saúde numa atitude transdisciplinar. 2012. 142 f. Dissertação (Mestrado) - Escola de Enfermagem Aurora de Afonso Costa (EEAAC) - Universidade Federal Fluminense (UFF). Niterói, 2012.

13 SOUZA, P.M.B.B. Protocolo de orientações de enfermagem para 0 paciente com insuficiência cardíaca à alta hospitalar. 2012. 127 p. Tese (Mestrado) - Programa de Pós-Graduação da Escola de Enfermagem Aurora de Afonso Costa, da Universidade Federal Fluminense, Niterói, Dezembro 2012.

14 CARMO, J.W.S. Gerenciamento do Cuidado Domiciliar do Idoso Após a Alta Hospitalar. 2014. 21f. Monografia (Especialização em Atenção Básica à Saúde) - Universidade Federal de Minas Gerais, Governador Valadares, 2014.

15 GANZELA, M. A alta hospitalar na avaliação de pacientes e cuidadores: uma revisão integrativa da literatura. Acta Paulista de Enfermagem. Ribeirão Preto. v. 21, n. 2, p. 351-355. Out. 2008. 